After recovering from this he never felt really well again, had various vague pains, thought to be rheumatic, and sensations as if his head were being drawn downward and forward. He had diplopia for several weeks before actually going to bed, and had also bluish mists before his eyes. He engaged a locum tenens apparently about January 20, and went to bed. The following day he was practically comatose, and had no recollection for several days after this. I saw him on February 25 with Dr. Randall, Wimbledon. He was then partially conscious, with double ptosis and defective eye movements. He gradually improved, and in two months was at work. I saw him about a year later. He had continued at work and was able to carry on without much difficulty. No defective ocular movements were present, but he complained of stiffness, and his aspect: was that of a patient with slight paralysis agitans without tremor. He has continued to improve steadily.

These cases, in which the affection was not severe-numbers 4 and 5-are, I think, of peculiar interest, for the symptoms were comparatively slight and the recovery was practically complete. The other cases are more marked, evidently the result of a more severe intoxication, but in all of them the eye symptoms were distinct and in some of the cases striking.

\title{
A CASE OF MELANOSARCOMA CONJUNCTIVAE BULBI
}

$\begin{array}{ccc}\text { L. K. WOLFF, } & \text { any } & \text { DR. H. T. DEEIMAN, } \\ \text { OCULIST BACTERIOLOGIST, FIRST } & & \text { DIRECTOR OF THE LABORATORY OF } \\ \text { ASSISTANT OF THE LABORATORY OF } & \text { THE ANTONI VAN LEEUNEN- } \\ \text { HYGIENE OF THE AMSTERDAM } & \text { HOEKHOUSE (NETHERLAND INSTITUTE } \\ \text { UNIVERSITY, } & \text { FOR CANCER RESEARCH). }\end{array}$

Although melanosarcoma of the interior of the eye is not very rare, similar tumours of the exterior are only exceptionally met with. As the latter tumours show considerable differences in their clinical progress when contrasted with the former, we consider that an elaborate account of the history of such a tumour should prove of value, especially as some general conclusions may be drawn from our account.

Our patient was born in 1881, and first came under observation in the autumn of 1916. She had noticed a small black spot the size of a pin's head on the left conjunctiva during the last ten years, and thought that it had become a little bigger in the last few years. Very recently a small red blister had appeared, and it was on account of this that she sought medical assistance. The patient has always been healthy and has had five healthy 
children. She is a brunette with dark hair, brown eyes, and dark complexion. She has many small moles on the body, as also have her sisters and her children. The report of the condition of the left eye is as follows: On the conjunctiva bulbi at the side of the cornea there is a small half round tumour. The surface is irregular and shining and about half the tumour is pigmented. It is only loosely attached to the subconjunctival tissue. There is a superficial stripe of pigment surrounding the tumour. The vessels supplying the tumour are tortuous and swollen (Fig. 1).

The question was, what to do with this case? Seeing that these tumours tend to recur locally after they have been radically removed (a point which we shall discuss later), the removal of the

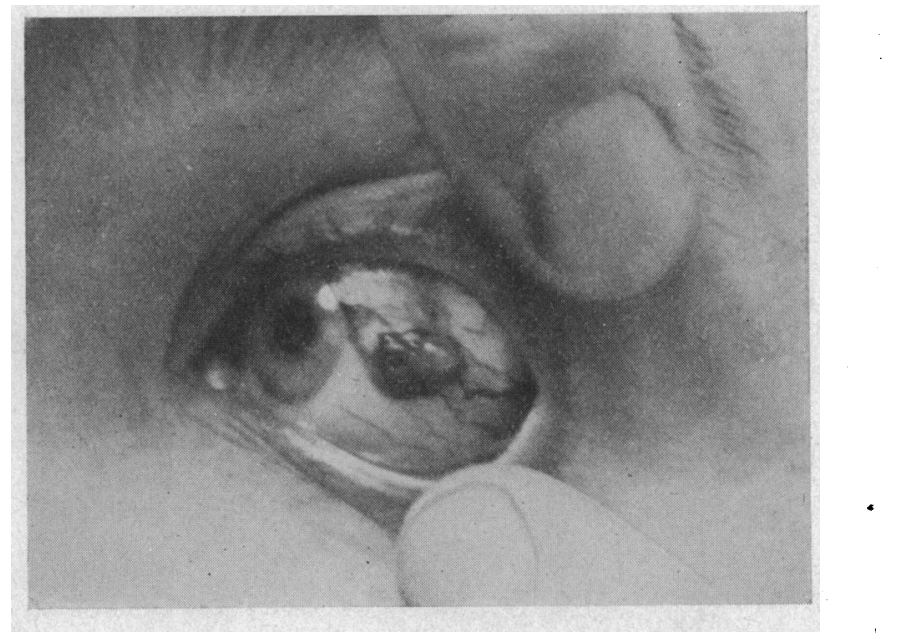

FIG. 1.

whole eye had to be considered, a very serious thing for a woman of thirty-five, especially as the functions of the eye were absolutely normal. Since in recent years $X$ ray therapy has proved of some value in such cases, we decided to remove the tumour alone and subsequently to expose the eye to this treatment, as we knew from Axenfeld's account that such exposure was not likely to injure the eye. We, therefore, removed the tumour with a wide strip of conjunctiva.

The anatomical examination of the tumour showed that macroscopically we have to do with a granular piece of tissue which is irregularly coloured black and brown; microscopically it appears to be covered on three sides with pavement epithelium. Under the epithelium there is a tumour tissue which reaches the surface in several places. Where this is the case the epithelium has become very thin. Especially in the deeper parts of the tumour 
the structure is markedly alveolar. The protoplasm of the cells, under higher magnification, is often indistinct, sometimes granular and sometimes frothy. The nuclei are often spindle-shaped. Where the alveolar structure becomes apparent the cells are often spindle-shaped. The situation of the pigment is of importance. This pigment is mainly found as granules of varying size lying in the stroma of the connective tissue between the formed cell alveoli. When in the alveoli it is generally to be found in the most external cells. In addition, irregularly outlined dark coloured cells are to be seen in small vessels (blood or lymph vessels). Since these are tumour cells that have extended beyond the section into the lumen, the vascular wall must have been

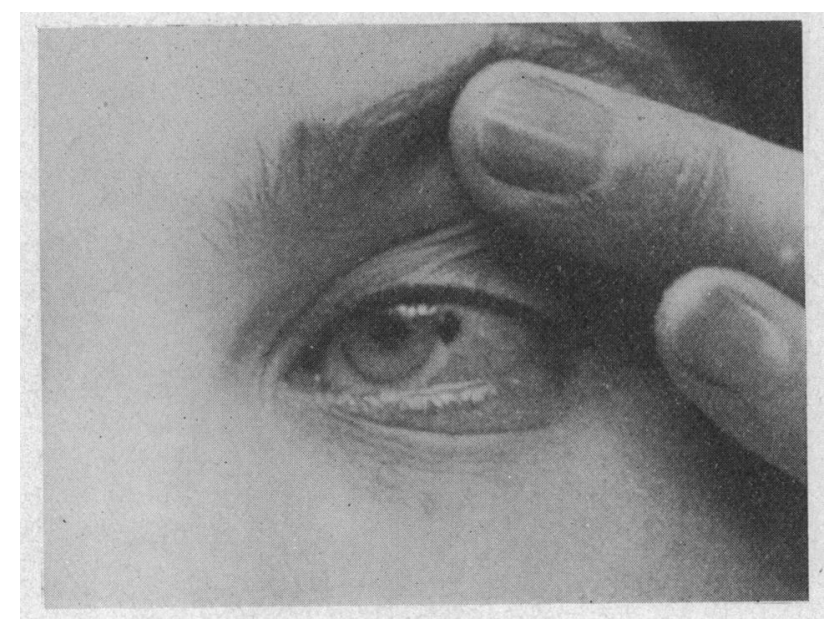

Fig. 2:

broken through. In other places where the external limitations of the formed cell groups consist of a flat layer of cells, it is impossible to decide whether we are dealing with a tumour adhesion which has been cut through vertically, or with divided vessels filled with tumour cells. Nuclear divisions are not very numerous, but are easily found. The microscopical diagnosis was: melanosarcoma (type, melanosarcoma alveolare). The preparations did not enable us to ascertain if the removal had been radical or not.

The subsequent history is as follows: the wound healed well in a few weeks, leaving the conjuuctiva slightly oedematous and light rose coloured. The operation wound was then exposed to a rather strong $\mathrm{X}$ ray treatment (in total $16 \mathrm{H}$. units were given, filtration $5 \mathrm{~mm}$. aluminium). A few months later the condition is as follows : there is a small stripe of pigment which ends horizontally in the place where the tumour had been. This stripe of pigment 
lies in a part which looks as if it might be scar tissue, and extends from the edge of the cornea some way on to the bulbar conjunctiva. After the first series of exposures no further $X$ ray treatment was given. The patient attended' regularly for inspection, and no change was noted during the rest of 1916 and the whole of 1917 till December. The attending physician thought that at that time there had been some increase in the width of the pigment stripe. In March, 1918, there was no doubt of this, as, with the aid of magnification, the pigment could be seen to have extended into the cornea, which had not been previously observed. The photograph (Fig. 2) shows the condition of the eye.

The problem of treatment now became a difficult one. There was no question of a tumour in the clinical sense of the word, and the intact condition of the corneal epithelium spoke against a tumour relapse. The eye performed its functions normally, and

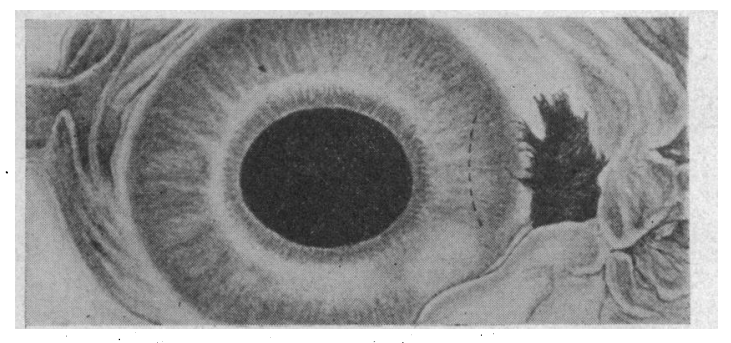

FIG. 3.

gave no trouble. Had we to dg with a simple melanosis, possibly due to the $\mathrm{X}$ ray exposure, or a remains of tumour pigment, or was there a question of a tumour itself ? After ample discussion we decided to remove the eye, because clinically it could not be said which of the two processes we had to deal with, and because, with this doubt, it was safer for the patient to think the worst. A prolonged $\mathrm{X}$ ray exposure was rejected, because the effect could not be controlled. The eye was removed in May, 1918. Recovery was uneventful, and the patient was subsequently fitted with an artificial eye. The condition of the eye after removal is better understood from Fig. 3 (after a drawing) than from any verbal description. Magnification shows that the peculiar pigmentation extends further than is represented in the drawing.

Macroscopical examination shows no other abnormalities, and . there are no over-filled blood-vessels as in the tumour that was first removed. The surface of the pigmented parts is smooth and shining. Horizontal sections were made through the pigmented part after imbedding in celloidin. Microscopically there appears to be a pigment focus in and under the epithelium. The greatest 
extension is at the corneo-scleral junction (Fig. 4). Here we find a focus of cells which under low magnification is specially conspicuous when compared with the cornea and sclera which are poorly supplied with cells but rich in fibrous tissue. Under high magnification some parts are seen to possess numerous capillary vessels, the spaces between which are filled up with irregularly formed pigment cells, mostly large and elongated. There are also many mononuclear and several plasma cells. The pigment cells are found directly under and in the epithelium, and also massed together in cavities at the expense of the epithelium. Locally the

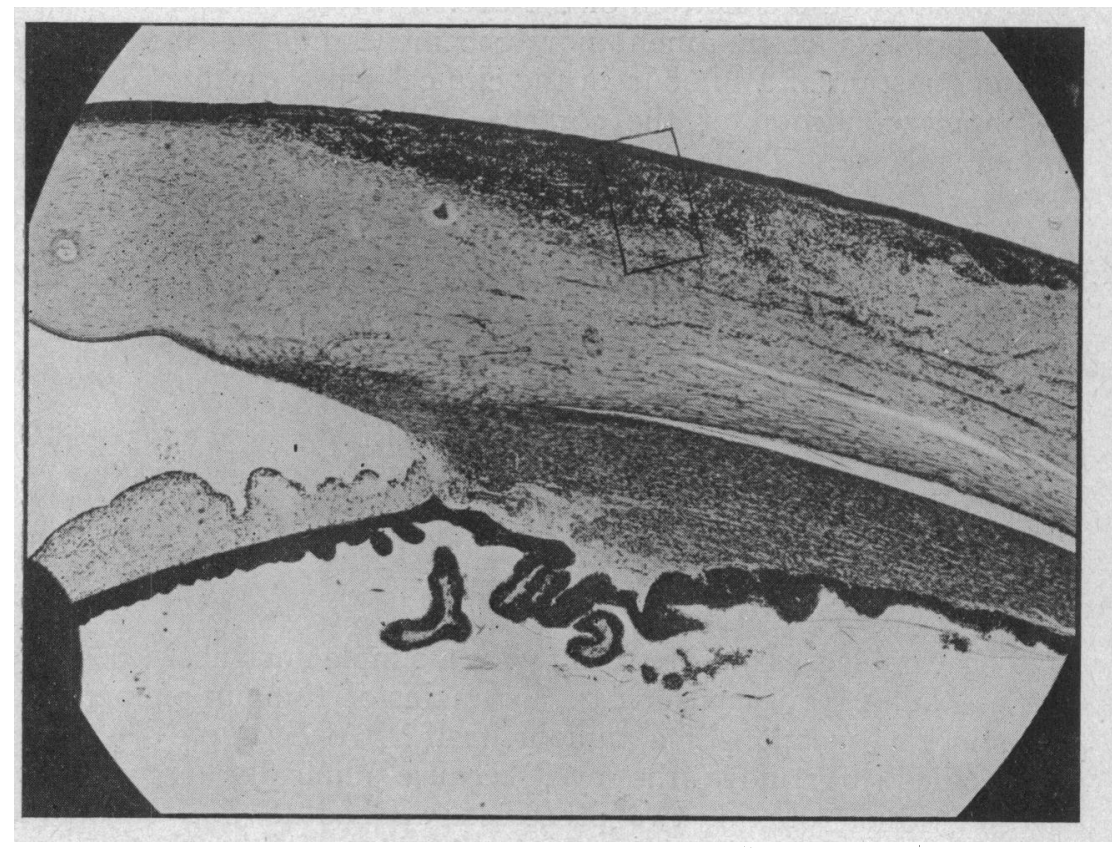

Fig. 4.

epithelium becomes very thin, and in some places it has disappeared entirely, to be replaced on the surface by a group of pigment cells. We do not feel able to decide that these spots may not have been artificially produced by removal of epithelium at the operation. Fluorescein never showed any staining. In the deeper layers of the focus of pigment cells we can see distinctly how the cells tend to arrange themselves along the course of the vessels (Fig. 5, from a drawing after a microphotograph). There is no distinct rupture of the vessels, but there is obliteration from the pressure of the cells. It is also noteworthy that some irregularly situated leucocytes are found in the lumen of the vessels. The pigment cells penetrate into the epithelium of the cornea and conjunctiva, 
and extend a considerable distance from the main focus. In the corneal epithelium they extend up to the line of dots as represented in Fig. 3. We also find pigment cells scattered here and there in the sclera. The fibres of the sclera, situated directly under the pigment focus, show a winding course.

What is the condition here, melanosis or tumour? We do not hesitate to decide upon the second. The infiltrating growth in the

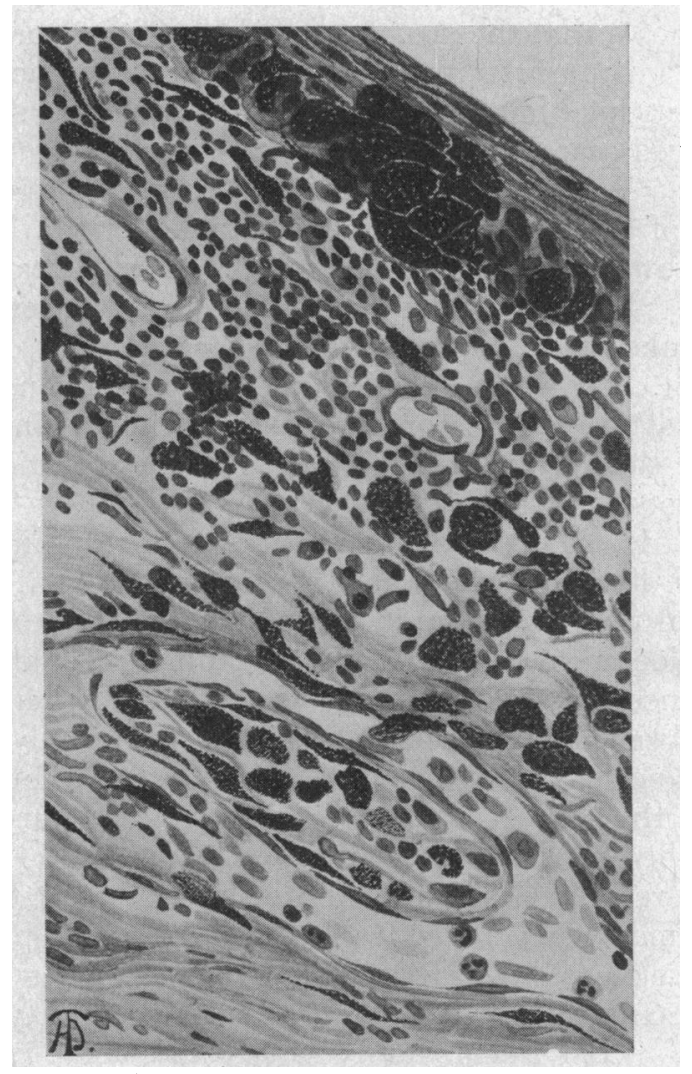

FIG. 5.

epithelium, the destruction of the epithelium, the vessel growth under the epithelium, and the arrangement of the cells along the vessels, show that we have to do here with a tumour relapse. Thus the removal of the eye is justified by the microscopical examination, since the deep pigment focus would have been out of reach of radical treatment.

The subsequent history of the case was as follows: in the autumn of 1919 , rather more than a year after the removal of the eye, the patient returned with a tumour in the temple, near the 
left eye. This was removed by an arched incision close to the orbit. The temporal muscle was cut through and partly removed. Healing was uneventful.

Pathological examination of the removed tissue shows an irregularly shaped mass, as large as a cherry, the surface of which is mostly formed of fat with a piece of muscle. Inside a harder mass can be felt. On the deeper side there is a sharply outlined greyish-white and rather hard tumour mass. Microscopical examination shows that the structure of this tumour corresponds with that of the tumour first removed, with this difference, that only a minimum amount of pigment can be found in the second growth after prolonged search.

Summary. We have the presence of a spot of pigment on the conjunctiva of the left eye for a considerable period. This suddenly begins to grow rapidly and is removed. $\mathrm{X}$ ray treatment follows the removal. In spite of the use of a powerful series of exposures recurrence takes place a year later in the situation of the original pigment spot. The eye is then removed. At a considerably later period a metastasis appears in the neighbourhood, almost free from pigment and easily removable. After the last operation the patient remains in good health.

Remarks. If we read the literature of these tumours we find that, in spite of their rarity, there is a certain amount of uniformity in the clinical process. The melanosarcomata of the interior of the eye are considered to be very malignant tumours, as evidenced from the appearance of widespread metastases even when the primary tumour has been removed soon after its discovery (liver metastases, general melanosis). The following condensed account from the literature of the subject illustrates the clinical course of melanosarcoma of the exterior of the eye.

Lagrange $^{1}$ recorded in 1901 twenty-three cases, taken from the literature, among which are two of his own. This shows the rarity of these cases. The extraocular tumours do not display the same malignant course as the intraocular variety. Although metastases do occur, such occurrence usually takes place at a later period and only in certain regions. On the other hand local recurrence after removal is frequent.

The limbus is considered a site of predilection for these tumours, especially the portion lying between the lids. They also, though very rarely, occur in other parts (bulbar conjunctiva, caruncle). Heller $^{3}$ gives an account of the localization. He gathered 80 cases from the literature and found, that, in so far as he could trace the seat, 37 cases originated from the limbus, 17 from other parts of the conjunctiva, 7 from the caruncle, and 1 from the sclera.

All statements agree that the growth is a very slow one. The tendency is for the tumours to grow outwards, and the patients 
usually seek advice on account of difficulty in closing and opening the eyes.

Relapse is frequently recorded after local removal. In many cases the patients say that formerly there had been a pigment spot in the situation of the tumour which suddenly developed in that situation (in the 80 cases quoted by Heller, this pigment spot existed no less than 36 times). Wolfrum ${ }^{4}$ examined the bulbar conjunctiva of normal eyes for the presence of pigment and found that it was of more frequent occurrence than a superficial examination would have led him to expect. Especially along the limbus pigment seemed to occur when looked for with sufficient magnification. Since the origin of these tumours may take place from these microscopic pigment spots as easily as from the larger, macroscopic, ones, it is not improbable that all these tumours start from conjunctival pigment; the patient, however, may not know of any previous pigment spot in the case where such spot was very minute.

In the cases recorded by Degering, Green, Erwing, and Sickerer a pigment spot at the site of the tumour had existed since youth. In another case a naevus had existed for ten years before the tumour. This was the case of a man of 22 who had a small yellow, slightly raised tumour on the eye for three or four years. It measured about $4 \mathrm{~mm}$. by $2 \mathrm{~mm}$., and was situated on the external side of the eye close to the limbus, in exactly the same place as the growth dealt with in this paper. It was transparent, and on the upper side a little stripe of brown pigment was visible. Some vessels led up to it and could also be recognized in the substance of the tumour. It had not grown and had caused no trouble. It was said to have followed inflammation of the eye (conjunctivitis ?). Two years later the condition was still the same. Three and a half years later the only change noted was the development of what looked like a local arcus senilis at the place where the growth touched the cornea. This was barely $1 \mathrm{~mm}$. wide, and seemed to have grown gradually a little wider. On this account the tumour was removed, under local anaesthesia, six and a quarter years after the first consultation, and ten years after it was first noticed. Healing was uneventful. Microscopically the epithelial layer was intact, under which there was a sharply outlined tumour mass, consisting mainly of larger and smaller groups of cells that were often of a light colour, and rich in protoplasm. The formed tumour alveoli were often very distinctly outlined, although there were also parts of the tumour more definitely sarcomatous in construction; at least there the stroma disappeared entirely, and the alveolar structure ceased. Again in places the structure resembled that of an endothelioma, with unattached cells arranged like an irregular tube. Here and there a small pigment focus existed (iron-free pigment), where more pointed and spindle-shaped cells, loaded with pigment, 
remained in the stroma, and where there was also granular pigment in the epithelioid cells.

Such cases strongly suggest the origin of melanosarcomata from pigmented naevi. The irregularly polymorphous structure of the melanosarcomata, the irregular pigmentation, etc., all look so much like the structure observed in a naevus that, involuntarily, we come to the conclusion that only very slight changes are necessary for a sarcoma to develop from a naevus.

For the time being, the problem of the origin of these tumours cannot be solved. As to the melanosarcomata, this question corresponds to that of the origin of the construction elements of the pigment naevi. So long as the schools of Unna and Ribbert do not agree about the origin of the naevus cells as they appear in the superficial cutaneous layers in moles; we think it is of little use to discuss the origin of the melanosarcomata in detail here. One can, however, imagine cases which have to be examined by a pathologist-anatomist, especially of the forepart of the eye, which could be of great importance for the solution of this question. For these tumours can be quickly diagnosed, and so we may expect here that even the very smallest turnours are brought for inspection (the varying sarcomatous and alveolar construction of these tumours make the solution of the question of origin, epithelium or mesenchyma, all the more difficult).

There have been many attempts to prove that these tumours originate from the pigment tumours of the conjunctiva bulbi and from the naevi.

Wolfrum made a study of this question, and he thinks he may infer from his slides that the naevus cells grow directly downwards from the epithelium cells. The reverse, however, viz., that the naevus cells come from below and attach themselves to the epithelium, may be deduced from his illustrations as well. Frick ${ }^{5}$ is right in indicating that Wolfrum's study has not made us much wiser as to this question. So the question of the origin of the melanosarcomata remains unsolved for the melanosarcomata of the conjunctiva bulbi, as well as for the origin of naevi of this organ or of all other organs.

Especially as regards the beginning of the growth, traumata have been thought to play a rôle. The superficial origin and the situation between the eyelids are used as arguments in favour of this theory. The patients frequently come with the complaint that something has got in the eye, after which the tumour began to grow. Lowenthal ${ }^{6}$ mentions in 1894 that among the small number of tumours which were described at that time, trauma appeared in the history of these swellings five times. Nevertheless, we read that both macroscopic and microscopic pigment spots frequently occur in the same part. Wolfrum 
points out that the pigment of the limbus and its surroundings especially occurs within reach of the opening between the lids-so the connection between trauma and the formation of tumours in this place a priori becomes less likely.

Finally, it is important to notice that the growth always develops towards the outside. A growth towards the inside of the eye seems to be very rare in these tumours. The growth then passes along the perforating anterior ciliary vessels. In one case sarcomatous cells were found in the canal of Schlemm (Parsons ${ }^{7}$ ). As to the latter, it is the real carcinomata which seem to disregard the anatomical borders. Carcinomata of the conjunctiva bulbi more frequently grow towards the inner eye, destroying the coats of the eye $\left(\right.$ de Vries $\left.{ }^{8}\right)$.

Besides, we were also struck by the descriptions in the literature saying that the metastases of these tumours are free from pigment.

Summarizing, we have here a case of a very rarely occurring form of melanosarcoma, which, however, as to its course is " typical," by which we mean :

1. Origin from a pigment spot.

2. Growth to the outside.

3. Relapse.

4. Slow metastasis.

The case has taught us that we may have to deal with a tumour relapse even when the clinical picture cannot be recognized as such. Then the physician is confronted with the difficult question of how to act. In such cases he will have to anticipate the worst danger and remove the eye. So in such a case we must not stop at local therapeutics, but remove the entire eye at once. The $\mathrm{X}$ ray therapeutics on which we had founded our hope had left us in the lurch here. But the $\mathrm{X}$ ray treatment has not done any harm to the eye. Clinically and microscopically we did not find any change that may be attributed to the exposures. This is of importance because it has appeared from the literature (which was not yet known in 1916) that the glioma eyes 'of Axenfeld all became cataractous; most probably the way of exposing to $\mathrm{X}$ rays in the Leeuwenhoekhouse was more careful, as some of Axenfeld's cases became cataractous within a period of one to one and a half years.

\section{LITERATURE.}

I. Lagrange, "Tumeurs de l'oeil," 1920.

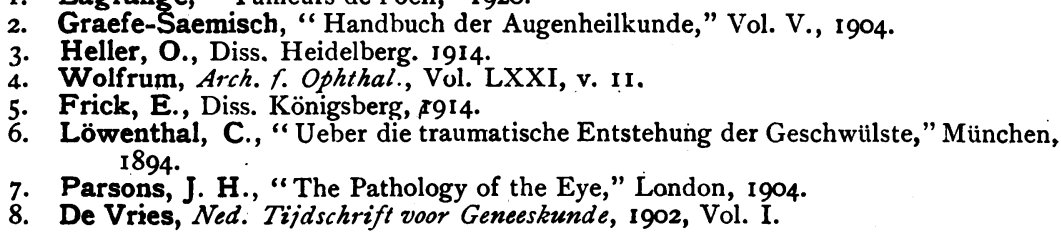
1894 .

7. Parsons, J. H., "The Pathology of the Eye," London, 1904.

8. De Vries, Ned. Tijdschrift voor Geneeskunde, 1902, Vol. I. 